\title{
Competing ultrafast intersystem crossing and internal conversion in the "channel 3" region of benzene
}

R.S. Minns, D.S.N. Parker, T.J. Penfold, G.A. Worth and H.H. Fielding

School of Chemistry, University of Southampton, Southampton UK SO17 1BJ. Email: R.Minns@soton.ac.uk

Please cite this paper as:

Physical Chemistry Chemical Physics, 2010, (12) 15607-15615

The publisher's version of this paper is available here:

http://dx.doi.org/10.1039/C001671C

\section{Related articles by Dr Russell Minns can be found below:}

T.J. Penfold, R. Spesyvtsev, O.M. Kirkby,; R.S. Minns,; D.S.N. Parker, H.H. Fielding, G.A. Worth, (2012) Quantum dynamics study of the competing ultrafast intersystem crossing and internal conversion in the "channel 3" region of benzene. Journal of Chemical Physics (137), 20 204310-24311 (doi:10.1063/1.4767054).

V.S. Petrovic, M. Siano, J.L. White, N. Berrah, C. Bostedt, J.D. Bozek, D. Broege, M. Chalfin, R.N. Coffee, J. Cryan, L. Fang, J.P. Farrell, L.J. Frasinski, J.M. Glownia, M. Guhr, M. Hoener, D.M.P. Holland, J. Kim, J.P. Marangos, T. Martinez, B.K. McFarland, R.S. Minns, S. Miyabe, S. Schorb, R.J. Sension, L.S. Spector, R. Squibb, H. Tao, J.G. Underwood, P.H. Bucksbaum. (2012) Transient X-Ray Fragmentation: Probing a Prototypical Photoinduced Ring Opening. Physical Review Letters 108, (25) 253006 (doi:10.1103/PhysRevLett.108.253006).

R.S. Minns, D.S.N. Parker, T.J. Penfold, G.A.Worth, H.H. Fielding, (2010) Competing ultrafast intersystem crossing and internal conversion in the "channel 3 " region of benzene. Physical Chemistry Chemical Physics 12 (48) 15607 (doi:10.1039/C001671C)

A.D.G. Nunn, R.S. Minns, R. Spesyvtsev, M.J. Bearpark, M.A. Robb, H.H. Fielding, (2010) Ultrafast dynamics through conical intersections and intramolecular vibrational energy redistribution in styrene. Physical Chemistry Chemical Physics 12 (48) 15751 (doi: 10.1039/COCP01723J)

D.S.N. Parker, R.S. Minns, T.J. Penfold, G.A. Worth, H.H. Fielding, (2009) Ultrafast dynamics of the S-1 excited state of benzene. Chemical Physics Letters 469 (1-3) 43 (doi:10.1016/j.cplett.2008.12.069) 


\title{
Competing ultrafast intersystem crossing and internal conversion in the "channel 3" region of benzene
}

\author{
R.S. Minns ${ }^{1}$, D.S.N. Parker ${ }^{1}$, T.J. Penfold ${ }^{2}$, G.A. Worth ${ }^{2}$ and H.H. Fielding ${ }^{1 \star}$ \\ ${ }^{1}$ Department of Chemistry, University College London, 20 Gordon Street, London \\ WC1H OAJ, UK \\ ${ }^{2}$ School of Chemistry, University of Birmingham, Birmingham B15 2TT, UK
}

\begin{abstract}
We report new, detailed, femtosecond time-resolved photoelectron spectroscopy experiments and calculations investigating the competition between ultrafast internal conversion and ultrafast intersystem crossing in electronically and vibrationally excited benzene at the onset of "channel 3". Using different probe energies to record the total photoelectron yield as a function of pump-probe delay we are able to confirm that $S_{1}, T_{1}$ and $\mathrm{T}_{2}$ electronic states are involved in the excited state dynamics. Time-resolved photoelectron spectroscopy measurements then allow us to unravel the evolution of the $\mathrm{S}_{1}, \mathrm{~T}_{1}$ and $\mathrm{T}_{2}$ components of the excited state population and, together with complementary quantum chemistry and quantum dynamics calculations, support our earlier proposal that ultrafast intersystem crossing competes with internal conversion [Chem. Phys. Lett. 46943 (2009)].
\end{abstract}

*Email: h.h.fielding@ucl.ac.uk 


\section{Introduction}

Absorption and fluorescence measurements of the first excited state of benzene have highlighted two energy regions which show dramatically different dynamics ${ }^{1-30}$. Upon irradiation with light in the wavelength range $245-260 \mathrm{~nm}$, benzene is seen to fluoresce ("channel 1") with a quantum yield of approximately 0.2 , while the remaining population undergoes intersystem crossing (ISC) (“channel 2") to a low lying triplet state; ${ }^{14,} 23,24$ both of these processes are slow compared to the timescale of molecular rearrangement, taking somewhere between 10-100 ns to occur. ${ }^{23,24}$ If the wavelength is decreased below $245 \mathrm{~nm}\left(>3000 \mathrm{~cm}^{-1}\right.$ excess energy in $\mathrm{S}_{1}$ ) a new, extremely rapid molecular process ("channel 3") causes the spectral line widths to broaden, the spectrum to become more diffuse and the fluorescence quantum yield to fall to zero for some vibrational modes. ${ }^{2}$ Linewidth measurements show strong mode dependence and indicate some lifetimes as short as a few hundred femtoseconds, the time scale of vibrational motion. ${ }^{2,}{ }^{12,} 22$ The origin of the dramatic change from fluorescent to non-radiative behavior at the onset of "channel 3" has been a source of considerable discussion since the original observations were made in the 1960s and 1970s. Of the explanations put forward, there are two that have received most attention: direct internal conversion (IC) back to the ground state, or isomerisation to one of the many valence isomers, followed by rapid IC to the ground state.

Following the first experiments reporting ultrafast non-radiative decay in the "channel 3" region by Callomon et $a l^{1}$, rotationally resolved spectra ${ }^{4,8,9,11,20,23,28}$ revealed that, for a given vibrational state, the lifetimes were strongly-dependent on the rotational quantum number. Below "channel 3", experiments by Rice and coworkers showed that the rate of ISC as a function of excess energy was fairly random; ${ }^{14,24}$ however, if the rate of ISC was plotted as a function of the number of quanta in a particular vibrational mode, a monotonic increase in the rate of ISC was observed. Nonetheless, the fastest nonradiative lifetimes observed were only tens of nanoseconds, five orders of magnitude slower than the lifetimes reported for "channel 3".

The rapid onset of "channel 3" is indicative of a barrier on the excited potential surface, either to a conical intersection leading to rapid radiationless decay, or towards other valence isomers from which rapid non-radiative decay can occur. Experiments by the Callomon group explored the possibility of other valence isomers being produced en route to the benzene ground state. ${ }^{3}$ Deuterium labeled benzene molecules were exposed to a broad bandwidth photolysis lamp. If prismane and benzvalene isomers had been produced on the pathway back to the ground state, the positions of the deuterium atoms 
would have been scrambled compared to their initial positions, which would have been observed in the absorption spectra of the products; however, no such scrambling was observed, suggesting that isomerisation, to prismane and benzvalene at least, was not the primary step in the non-radiative decay in the “channel 3” region.

The most widely accepted explanation for the rapid non-radiative lifetime seen in the "channel 3" region has been that rapid IC occurs through a conical intersection between the ground and first excited state that lies along the reaction co-ordinate linking benzene and prefulvene, a biradical species with a non-planer geometry. ${ }^{31}$ The conical intersection is located just the other side of a barrier, near a saddle point, which acts as an efficient funnel with high sides forcing the molecule towards the conical intersection and preventing the molecule escaping along different reaction coordinates. The electronic energy of $S_{1}$ is transferred to vibrational energy in the ground state via a large vibronic coupling leading to extremely hot ground state molecules. Recent femtosecond pumpprobe photoionisation measurements confirmed lifetimes $<300$ fs at some excitation energies which, as the authors suggested, are compatible with IC. $^{29}$ However, experiments simply measuring the integrated ionization cross-section as a function of pump-probe delay cannot differentiate between IC, ISC or isomerisation.

The key to developing a detailed picture of the "channel 3" dynamics in the prototypical organic molecule benzene, and to developing a detailed understanding of the excited state dynamics of any molecule, is to measure the electronic and nuclear composition of the total wave function of the molecule as it evolves. In practice, because the electronic and nuclear motions are strongly coupled, this is not trivial; however, time-resolved photoelectron spectroscopy (TRPES) is emerging as an extremely valuable tool for following excited state non-adiabatic processes in molecules. ${ }^{32-35}$ First, an ultrafast laser pulse (pump) prepares the molecule in an electronically and vibrationally excited nonstationary state, and then, a subsequent ultrafast laser pulse (probe) ionises the molecule and projects the evolving molecular wave function onto the molecular ionization continuum. By measuring the resulting photoelectron spectrum as a function of pumpprobe delay, one can gain some insight into the electronic and geometric changes along the reaction coordinate. TRPES has been employed to monitor wave packet dynamics in simple diatomic molecules, ${ }^{36}$ to follow IC in polyenes, ${ }^{37}$ photodissociation, $^{38-40}$ intramolecular vibrational energy redistribution (IVR) ${ }^{41}$ and ISC. ${ }^{40,}{ }^{42}$ However, when there is a high density of electronically excited states correlating to the same electronic state of the cation, sometimes referred to as a type II Koopman correlation, it can be extremely challenging to decompose the evolving wavefunction into its constituent parts. 
Recent TRPES experiments in our group ${ }^{30}$ and frequency-resolved photoelectron spectroscopy experiments by de Groot and coworkers ${ }^{43}$ have shown that it is possible to determine the composition of the excited state wavefunctions of polyatomic molecules with a high density of electronically excited states by tuning the probe through the ionization continuum and recording photoelectron spectra using a higher energy probe pulse.

This paper, builds on our earlier work reporting a competition between ultrafast internal conversion and ultrafast intersystem crossing in the "channel 3 " region of benzene ${ }^{30}$. In this more detailed investigation, we demonstrate how plotting the integrated photoelectron yields as a function of pump-probe delay for different probe wavelengths allows us to identify which regions of the photoionisation continuum are associated with ionization from the $S_{1}, T_{1}$ and $T_{2}$ electronic states. We then investigate the energy flow between these different components using time-resolved photoelectron spectroscopy. Calculations of the spin-orbit coupling strength at key points on the excited potential energy surface support our proposal that ultrafast intersystem crossing competes with internal conversion. Quantum dynamics calculations of the evolution of the excited state population are in excellent agreement with the experimental observations.

\section{Experiment}

The experimental approach is illustrated schematically in figure 1. Immediately after excitation by the pump pulse, the total excited state wavefunction is a linear combination of vibronic wavefunctions, $\psi_{i}(q) e^{-i E t / \hbar}$, integrated over the envelope of the electric field, $\varepsilon(E)$ :

$$
\Psi(t)=\int d E \varepsilon(E) \sum_{i, q} c_{i}^{q}(t) \psi_{i}(q) \exp (-i E t / \hbar),
$$

where $i$ labels the diabatic electronic states $\mathrm{S}_{1}\left({ }^{1} \mathrm{~B}_{2 \mathrm{u}}\right), \mathrm{T}_{1}\left({ }^{3} \mathrm{~B}_{1 \mathrm{u}}\right), \mathrm{T}_{2}\left({ }^{3} \mathrm{E}_{1 \mathrm{u}}\right)$ or $\mathrm{S}_{0}\left({ }^{1} \mathrm{~A}_{1 \mathrm{~g}}\right), q$ labels the vibrational modes and $c_{i}^{q}(t)$ are the time-dependent amplitudes. Each of the excited states, $S_{1}, T_{1}$ and $T_{2}$, has the molecular orbital configuration $\left(a_{2 u}\right)^{2}\left(e_{1 g}\right)^{3}\left(e_{2 u}\right)^{1}$ and correlates, upon removal of the electron from the highest energy $\mathrm{e}_{2 \mathrm{u}}$ orbital, to the ground electronic state of the cation, $\mathrm{D}_{0}\left({ }^{2} \mathrm{E}_{1 \mathrm{~g}}\right)$. The photoelectron spectrum of each of these electronic states can, in principle, be distinguished because they have different vibrational overlap integrals between their vibrational wavefunctions and those of the ground electronic state of the cation; however, in practice, the bands are strongly overlapping and therefore not trivial to disentangle. The improvement in the work presented in this paper 
is that the energy of the probe laser is scanned through the ionization continuum to determine which electronic states are involved in the excited state dynamics and where they are projected into the photoionisation continuum. We then unravel the evolution of the different electronic components of the excited state population from the photoelectron spectra and compare with quantum dynamics calculations. This is perhaps best explained by looking at figure 1. For a given excitation energy, $E_{\mathrm{vib}}\left(S_{1}\right)<E_{\mathrm{vib}}\left(T_{2}\right)<E_{\mathrm{vib}}\left(T_{1}\right)$, where $E_{\text {vib }}$ represents excess vibrational energy. For rigid molecules that only undergo small displacements, there is a tendency for the vibrational quantum number to be preserved during photoionisation. ${ }^{44}$ Thus, to a first approximation for benzene, one would expect the threshold for ionization out of $T_{1}$ to be higher than that from $T_{2}$ which, in turn, is slightly higher than that for $\mathrm{S}_{1}$.

The experimental apparatus consists of a femtosecond laser system, molecular beam and velocity map imaging spectrometer. The commercial femtosecond laser system consists of an ultrafast oscillator and amplifier producing a $1 \mathrm{kHz}$ train of $2.5 \mathrm{~mJ}$ pulses of $35 \mathrm{fs}$ duration and a central wavelength of $795 \mathrm{~nm}$. Approximately $70 \%$ of the light from the amplifier pumps two optical parametric amplifiers (OPAs) to generate tunable infrared (IR) radiation between $1200 \mathrm{~nm}$ and $2000 \mathrm{~nm}$. The signal from each OPA is then mixed with the residual pump light to produce visible light between $470 \mathrm{~nm}$ and $600 \mathrm{~nm}$ which is subsequently frequency doubled to produce tunable light around $250 \mathrm{~nm}$. The pulse energy of each of the UV beams is attenuated to $<1 \mu \mathrm{J}$ to reduce unwanted multiphoton ionization. For all of the experiments described in this paper the pump pulse is centered at $243 \mathrm{~nm}$ and takes us to the transition region in $S_{1}$ with $3070 \mathrm{~cm}^{-1}$ excess vibrational energy, at the onset of 'channel 3'. The central wavelength of the probe pulse is varied between 235 and $260 \mathrm{~nm}$. The temporal delay between the pump and probe pulses is set by a computer controlled mechanical translation stage. Both beams are combined at a thin dichroic mirror and focused collinearly into a molecular beam of benzene, in the source region of a photoelectron imaging spectrometer, using a $250 \mathrm{~mm}$ fused silica lens. The cross-correlation of the pump and probe pulses is $175 \pm 10 \mathrm{fs}$, measured by non-resonant ionization of NO.

The photoelectron imaging spectrometer consists of two differentially pumped chambers separated by a $1 \mathrm{~mm}$ skimmer. The source chamber houses the molecular beam (described below) and is pumped by a $2000 \mathrm{l} / \mathrm{s}$ maglev turbomolecular pump maintaining a base pressure of $8 \times 10^{-8}$ mbar when the molecular beam is off and approximately $5 \times 10^{-6}$ mbar when the molecular beam is running. The detection chamber contains a velocity map imaging (VMI) spectrometer and a liquid nitrogen cold trap and is pumped 
by a $1000 \mathrm{l} / \mathrm{s}$ turbomolecular pump maintaining a background pressure of $9 \times 10^{-8} \mathrm{mbar}$, which rises to $1 \times 10^{-7}$ mbar when the molecular beam is running. The VMI spectrometer is based on the Eppink and Parker design ${ }^{45}$ and consists of a set of three polished parallel field plates (repeller, extractor, ground) separated by $15 \mathrm{~mm}$. The extractor voltage was approximately 0.7 of the repeller voltage, which was set to $1.45 \mathrm{kV}$ for this work. The photoelectrons are accelerated through a $40 \mathrm{~cm}$ field-free region (surrounded by a layer of $\mu$-metal shielding) and collected by a commercial imaging detector (Photek) comprising a pair of microchannel plates backed by a phosphor coated optical fibre bundle. A CCD camera detects the light transmitted through the fibre bundle and the images are collected on a computer. In the path of both the pump and probe beams are separate mechanical shutters. For each pump-probe delay, we collect pump only, probe only and pump + probe images, in sequence, for 20 seconds each. This is repeated many times for a total of 1-2 hours for each time step. This helps reduce the effects of any long term drift in power due to laser instabilities. The pump only and probe only signals are subtracted from the pump + probe signal to eliminate single colour contributions from the images. Photoelectron spectra are recovered from the raw images using the image inversion program pBasex. ${ }^{46}$

Experiments were performed using a continuous effusive molecular beam of benzene created by continuously expanding liquid benzene (Aldrich 99\% ACS reagent grade, used without further purification) through a $50 \mu \mathrm{m}$ diameter nozzle held at room temperature. The vapour pressure of benzene at room temperature is $~ 110$ mbar which is sufficient to produce a stable effusive molecular beam.

The pump and probe intensities were kept well below $10^{11} \mathrm{~W} \mathrm{~cm}^{-2}$ to avoid multiphoton ionisation. Direct two-photon ionisation from the ground state is known to compete with 1+1 resonant multiphoton ionisation via $\mathrm{S}_{1} \cdot{ }^{47}$ As a check, we performed a number of measurements to confirm that the photoelectrons were not the result of unwanted multiphoton processes. The observed photoelectron count, $I \propto F^{n}$, where $F$ is the laser fluence and $n$ is the number of non-resonant photons involved in the ionisation process. In figure $2, \ln (I)$ is plotted against $\ln$ (pulse energy) - these measurements were performed using slightly higher pulse energies than those used in the pump-probe experiments, which were below the sensitivity of our power meter, and therefore provide an upper limit for $n$. For pulses centered at $254 \mathrm{~nm}$ and $235 \mathrm{~nm}, n=1.0 \pm 0.1$ and $n=1.2 \pm 0.2$, respectively and therefore we are confident that our experimental observations can be interpreted in terms of single-photon processes only.

\section{Results}




\subsection{Integrated photoelectron yield}

For all the results described in this paper the pump wavelength is $243 \mathrm{~nm}$, which corresponds to excitation with $3070 \mathrm{~cm}^{-1}$ excess vibrational energy above the zero-point energy level in the $\mathrm{S}_{1}$ potential ${ }^{7}, 29$, i.e. at the onset of 'channel 3'.

In figure 3, the integrated photoelectron yield for excitation of benzene in an effusive molecular beam is plotted as a function of the delay between the pump and probe lasers for three different probe wavelengths: $260 \mathrm{~nm}$ (lower trace), $254 \mathrm{~nm}$ (middle trace) and $235 \mathrm{~nm}$ (upper trace), corresponding to excitation into the ionization continuum approximately $0.6 \mathrm{eV}, 0.7 \mathrm{eV}$ and $1.1 \mathrm{eV}$ above $\mathrm{D}_{0}$, respectively.

When the probe wavelength is $260 \mathrm{~nm}$, the integrated photoelectron yield can be fitted to the sum of a biexponential decay and a sinusoidal oscillation with period $T=1.2 \pm 0.15$ ps,

$$
I(t)=\left[c_{\mathrm{f}} \exp \left(-t / \tau_{\mathrm{f}}\right)+c_{\mathrm{s}} \exp \left(-t / \tau_{\mathrm{s}}\right)\right]+c_{\text {osc }} \cos (2 \pi t / T) .
$$

$\tau_{\mathrm{f}}$ is the lifetime (1/e) of the fast component, $\tau_{\mathrm{s}}$ is the lifetime of the slow component, and $c_{\mathrm{f}}+c_{\mathrm{s}}+c_{\text {osc }}=1$. During the first $500 \mathrm{fs}$, approximately $60 \%$ of the excited state population moves rapidly away from the "ionization window" - the region of the excited state potential energy surface where it can be ionized by the probe pulse. Approximately $7 \%$ of the initially excited state population then returns and oscillates in and out of the ionization window with a period of $1.2 \mathrm{ps}$. The oscillation can be rationalized in terms of equation (1) by recognizing that the component wave functions are linear combinations of optically "bright" $\left(c_{i}^{q}(0) \neq 0\right)$ and "dark" $\left(c_{i}^{q}(0)=0\right)$ components (figure 4$)$ and that there must be two components within the excited state wave packet $(k=1,2)$,

$$
\psi^{(k)}=\left[c_{\text {bright }}^{(k)}(t) \phi_{\text {bright }}^{(k)}+c_{\text {dark }}^{(k)}(t) \phi_{\text {dark }}^{(k)}\right] \exp \left(-i E_{k} t / \hbar\right),
$$

with an energy separation $\left|E_{1}-E_{2}\right|$ and beat period $T=2 \pi /\left|E_{1}-E_{2}\right|$ (in atomic units). The maxima in the oscillations correspond to population in the "bright" components, which can be ionized by the $260 \mathrm{~nm}$ probe laser pulse, and the minima correspond to the "dark" components, which cannot be ionized by the $260 \mathrm{~nm}$ probe laser pulse. The energy separation must be $28 \mathrm{~cm}^{-1}$.

Increasing the energy of the probe laser pulse so that it excites higher into the ionization continuum results in a gradual decrease in the amplitude of the oscillation until eventually, when the probe reaches $254 \mathrm{~nm}$, the oscillation almost disappears altogether. Increasing the energy of the probe laser pulse increases the width of the ionization window. Both the bright and dark components can be ionized by the $254 \mathrm{~nm}$ probe laser 
pulse. The decay of the photoionisation cross-section can now be fitted to a simple biexponential function,

$$
I(t)=c_{\mathrm{f}} \exp \left(-t / \tau_{\mathrm{f}}\right)+c_{\mathrm{s}} \exp \left(-t / \tau_{\mathrm{s}}\right)
$$

Approximately $50 \%$ of the excited state population has moved out of the ionization window during the first 500 fs. The remaining excited state population decays very slowly, on a timescale of tens of picoseconds (the timescale of intramolecular vibrational redistribution).

Increasing the energy of the probe laser pulse further, exciting even higher into the continuum, does not have any effect on the overall appearance of the decay curve until the probe wavelength reaches $\sim 250 \mathrm{~nm}$ when there is a sudden increase in the amplitude of the slow component of the biexponential, corresponding to the ionization window opening up. ${ }^{30}$ For probe wavelengths in the range $250-235 \mathrm{~nm}$, approximately $30 \%$ of the excited state population moves out of the ionization window during the first $500 \mathrm{fs}$ and the remaining $70 \%$ of the excited state population decays very slowly.

\begin{tabular}{cccccc}
\hline$\lambda_{\text {probe }} / \mathrm{nm}$ & $\tau_{\mathrm{f}}$ & $\tau_{\mathrm{s}}$ & $c_{\mathrm{f}}$ & $c_{\mathrm{s}}$ & $c_{\text {osc }}$ \\
\hline 235 & $230 \pm 40 \mathrm{fs}$ & $1.7 \pm 0.5 \mathrm{~ns}$ & $0.32 \pm 0.03$ & $0.68 \pm 0.03$ & - \\
254 & $220 \pm 20 \mathrm{fs}$ & $11.5 \pm 1.0 \mathrm{ps}$ & $0.52 \pm 0.02$ & $0.48 \pm 0.01$ & - \\
260 & $141 \pm 15 \mathrm{fs}$ & $8.3 \pm 0.6 \mathrm{~ns}$ & $0.60 \pm 0.03$ & $0.37 \pm 0.01$ & $0.03 \pm 0.005$ \\
\hline
\end{tabular}

Table 1 The decay curves in figure 3 can be fitted to the functions in equations (2) and (4). This table lists the $1 / e$ lifetimes of the fast and slow components of the biexponential function, $\tau_{\mathrm{f}}$ and $\tau_{\mathrm{s}}$, and the coefficients, $c_{\mathrm{f}}, c_{\mathrm{s}}$ and $c_{\mathrm{osc}}$.

\subsection{Time-resolved photoelectron spectra}

From section 3.1, it becomes clear that to track the evolution of the excited state population across as much of the excited potential energy surface as possible requires the highest energy probe $(235 \mathrm{~nm})$. Time-resolved photoelectron spectra recorded using the $235 \mathrm{~nm}$ probe are presented in figure 5.

In order to see the changes in different regions of the photoelectron spectra more clearly, we present a set of difference-spectra in figure 6: the individual photoelectron spectra are scaled so that they all have the same integrated area (i.e. the biexponential decay in the integrated photoelectron signal is effectively removed) and the $t=0$ photoelectron 
spectrum is subtracted from all the other spectra. Regions of the photoelectron spectrum where we see a relative increase in photoelectron counts show up as peaks (warm colours) and regions where we observe a relative decrease in photoelectron counts appear as troughs (cool colours). The $t=0$ reference spectrum is displayed at the top of the difference-plots, and regions of particular interest are highlighted by red horizontal arrows.

Integrating across 1.25-1.35 eV $\left(a_{1}\right)$ and 1.13-1.23 eV $\left(a_{2}\right)$, results in a pair of cosine waves with period $1.2 \mathrm{ps}$ (the sums of these two components are plotted as red circles in figure 7). This is the same 1.2 ps oscillation observed in the integrated photoelectron signal obtained with the lowest energy $(260 \mathrm{~nm})$ probe. These higher kinetic energy electrons are assumed to arise following ionization of excited state population in $S_{1}$ (figure 1). Integrating across $0.75-0.95 \mathrm{eV}(b)$ results in a sine wave with period $1.2 \mathrm{ps}$ (green diamonds in figure 7). These slightly lower kinetic energy electrons are assumed to arise from ionization of excited state population in $T_{2}$ (as well as $S_{1}$ ) which lies just slightly higher in energy that $\mathrm{S}_{1}$ (figure 1). The amplitude of the oscillation in $a_{1}+a_{2}$ is equal to the amplitude of the oscillation in $b$ and these two oscillations are exactly $\pi$ out of phase with one another: this can be interpreted as a simple wave packet motion between $\mathrm{S}_{1}\left(a_{1}+a_{2}\right)$ and $\mathrm{T}_{2}(b)$. The two components $a_{1}$ and $a_{2}$ are separated by $\sim 0.12 \mathrm{eV}$ which, within experimental resolution, is the frequency of the breathing mode of the benzene cation. ${ }^{23}$

Finally, integrating across $0.35-0.45 \mathrm{eV}(c)$, yields a rise in the photoelectron signal at around $600 \mathrm{fs}$ (half the $1.2 \mathrm{ps}$ oscillation period). The integrated data points are plotted as blue triangles in figure 7 and are attributed to ionization of excited state population in $\mathrm{T}_{1}$ (figure 1) as well as $\mathrm{S}_{1}$ and $\mathrm{T}_{2}$.

\section{Discussion}

Using different ionization wavelengths to record the integrated photoelectron yield as a function of pump-probe delay (section 3.1), we have been able to identify where in the ionization continuum we must look to find signatures of the $S_{1}, T_{1}$ and $T_{2}$ excited states of benzene. We can then monitor the flow of energy between $S_{1}, T_{1}$ and $T_{2}$ using timeresolved photoelectron spectroscopy. Our results suggest that with just over $3000 \mathrm{~cm}^{-1}$ excess energy in $S_{1}$, approximately $30 \%$ of the excited state population undergoes rapid internal conversion to $\mathrm{S}_{0}$, around $20 \%$ of the excited state population undergoes rapid intersystem crossing to $\mathrm{T}_{1}$ and approximately $10 \%$ of the excited state population oscillates backwards and forwards between $S_{1}$ and $T_{2}$. This interpretation challenges the 
widely accepted view that intersystem crossing is negligibly slow compared to the timescale of internal conversion and therefore needs justifying.

Ultrafast intersystem crossing has been observed in a number of systems where the spinorbit coupling (SOC) is enhanced by the presence of a heavy atom or nitro group, in such cases lifetimes of just 100 fs have been observed. ${ }^{48,}{ }^{49}$ Benzene is distinct from these molecules in that it is a simple hydrocarbon with no obvious mechanism for such a large SOC. In fact, the SOC between $\mathrm{S}_{1}\left({ }^{1} \mathrm{~B}_{2 \mathrm{u}}\right)$ and $\mathrm{T}_{2}\left({ }^{3} \mathrm{E}_{1 \mathrm{u}}\right)$ at the Franck-Condon (FC) geometry is zero by symmetry, while the $\mathrm{S}_{1}\left({ }^{1} \mathrm{~B}_{2 \mathrm{u}}\right) / \mathrm{T}_{1}\left({ }^{3} \mathrm{~B}_{1 \mathrm{u}}\right)$ coupling is tiny leading to the relatively long non-radiative lifetimes of the vibrational states below the onset of "channel 3". Ultrafast intersystem crossing therefore appears unlikely unless the SOC drastically increases at geometries that become accessible as the excitation energy is increased. In a recent paper Zilberg ${ }^{50}$ used single point $a b$-initio calculations to show that SOC can increase significantly at the $S_{1} / S_{0}$ conical intersection. Worth and Penfold ${ }^{51}$ have used extensive calculations to show that the SOC not only rises along the prefulvene coordinate but also along various normal modes of vibration. Even so, the SOC remains small, of the order of a few $\mathrm{cm}^{-1}$.

To explore the energy flow in detail, a model vibronic Hamiltonian containing two coupled singlet states, $\mathrm{S}_{0}\left(\mathrm{~A}_{1 \mathrm{~g}}\right), \mathrm{S}_{1}\left({ }^{1} \mathrm{~B}_{2 \mathrm{u}}\right)$ and two triplet states, $\mathrm{T}_{1}\left({ }^{3} \mathrm{~B}_{1 \mathrm{u}}\right), \mathrm{T}_{2}\left({ }^{3} \mathrm{E}_{1 \mathrm{u}}\right)$ was prepared. The parameters for this model were obtained by a fit to ab-initio points at CASPT2 level calculated at various points on the potential energy surface. Figure 8A shows the cut along the important "prefulvene vector" which leads from the FC point to the $\mathrm{S}_{1} / \mathrm{S}_{0}$ conical intersection. A five-state singlet model using the same approach can been found $\mathrm{in}^{52}$. The SOC values were calculated with Molpro $^{53}$, using both $(6,6)$ and $(10,10)$ active spaces and Roos(3s2p1d/2s) basis set. The SOC was calculated along all of the normal modes, the important combination modes and the prefulvene vector. Zeroth, first and second order vibronic terms were all included. At equilibrium, a seemingly insignificant coupling exists between $\mathrm{S}_{1} / \mathrm{T}_{1}$ of $0.07 \mathrm{~cm}^{-1}$. The vibronic terms are calculated from the gradient of the couplings along each normal mode. There are significant first order vibronic terms along the modes which contain out of plane C-H bends, this is because the out of plane motion allows mixing between the $\sigma$ and $\pi$ orbitals providing a mechanism for SOC. Second order vibronic terms are most significant along $v_{4}, v_{6}$ and $v_{16}$. These modes are important in leading to the $\mathrm{S}_{1} / \mathrm{S}_{0}$ conical intersetion and are therefore vital to the Hamiltonian. The vibronic effects can increase the SOC by almost two orders of magnitude and values between 3.5 and $4.5 \mathrm{~cm}^{-1}$ are found along out of plane C-H bending modes. At the $\mathrm{S}_{1} / \mathrm{S}_{0}$ conical intersection the SOC between $\mathrm{S}_{1}$ and 
$\mathrm{T}_{1}$ has increased to $1.5 \mathrm{~cm}^{-1}$ using the $(6,6)$ active space and $2.0 \mathrm{~cm}^{-1}$ using the $(10,10)$ active space. The full details of the SOC used in the model will be published in more detail elsewhere. $^{51}$

Quantum dynamics calculations to determine the evolution of excited state population were performed using the Heidelberg MCTDH package ${ }^{54}$ with a model containing 8 normal mode degrees of freedom $\left(v_{1}, v_{4}, v_{6}, v_{8}, v_{9}, v_{14}, v_{15}\right.$ and $\left.v_{16}\right)$, two singlet states and two triplet states $\left(\mathrm{T}_{1}\right.$ and $\left.\mathrm{T}_{2}\right)$. Figure $8 \mathrm{~B}$ shows the diabatic state populations of the singlet and triplet states in the first 2000 fs. The blue line corresponds to the population of $S_{1}$ in the Franck-Condon region, i.e. minus the population which goes over the barrier to the $S_{1} / S_{0}$ conical intersection (see figure $8 \mathrm{~A}$ ). This corresponds to the photoelectron data using the $254 \mathrm{~nm}$ probe wavelength, which is only able to ionise wavepacket in the Franck-Condon region. The green line is the population of the entire $\mathrm{S}_{1}$ state and the red line is the population of $S_{1}$ plus the triplet populations, corresponding to the photoelectron data using the $235 \mathrm{~nm}$ probe pulse. Figure $8 \mathrm{~B}$ inset shows the diabatic state populations of only the triplet states in the first 2000 fs. The green line represents the $T_{2}$ population and the red line the $\mathrm{T}_{1}$ population. These states are coupled by a strong pseudo-JahnTeller interaction and so population crossing into $T_{2}$ quickly crosses further to $T_{1}$.

Comparing these dynamics to the experimentally obtained data shows excellent agreement and the theory clearly supports the idea that a significant portion of the diabatic state population is transferred from $S_{1}$ to $T_{2}$ followed by rapid IC from $T_{2}$ to $T_{1}$. In both cases, the fast decay is apparent for the first $500 \mathrm{fs}$. After 500 fs there is around $55 \%$ of the wavepacket in the Franck-Condon region of $S_{1}$, and $70 \%$ of the wavepacket in the $S_{1}$ and triplet states, this is almost identical to the experimental results. Unlike the experiments, after 500 fs the wavepacket continues to decay out of $S_{1}$. This can be attributed to the reduced dimensionality of the model. The population of the FrankCondon region of $S_{1}$ (blue) shows fast oscillations throughout the simulation attributable to the breathing mode motion. There is a clear maximum in the oscillations around $1.2 \mathrm{ps}$ which correlates with the wavepacket re-focusing along the prefulvene coordinate. However in this simple model no oscillation with this period is seen in the state populations.

\section{Summary}

Time-resolved photoelectron spectroscopy and pump-probe ionisation measurements have been employed to study the ultrafast dynamics of benzene at energies where the onset of channel 3 is observed. The ultrafast decay previously attributed to IC through a 
conical intersection is now realised to be due to competing IC and ISC processes occurring on similar femtosecond timescales. The relative proximity of the singlet-triplet crossing to the Franck-Condon region of benzene allows for efficient ISC at the expense of IC at a more distant conical intersection. As well as the fast decay to the triplet a repeated flipping between singlet and triplet multiplicities is observed.

\section{Acknowledgements}

The authors would like to thank J. H. Calloman, M. A. Robb and M. J. Bearpark for useful discussions. Financial support for this work was provided by the EPSRC, the Ramsay Trust (R. S. M.) and UCL.

\section{Figure Captions}

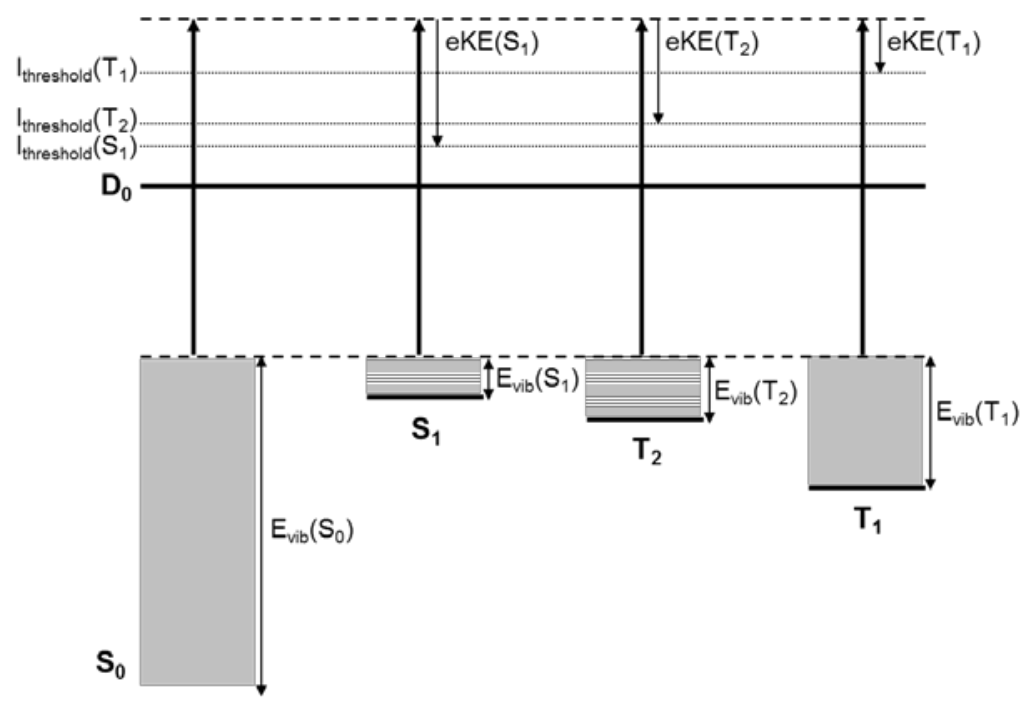

Figure 1

\section{Figure 1}

Schematic energy level diagram showing the two lowest singlet $\left(\mathrm{S}_{0}, \mathrm{~S}_{1}\right)$ and triplet $\left(\mathrm{T}_{1}\right.$, $T_{2}$ ) states of benzene and the ground electronic doublet $\left(\mathrm{D}_{0}\right)$ of the benzene cation. In the experiments described in this paper, benzene is excited with $\sim 3000 \mathrm{~cm}^{-1}$ excess energy in $\mathrm{S}_{1}$, marked by the lower horizontal dashed line. There is an approximate propensity for the vibrational quantum number to be conserved during photoionization; therefore, since 
$E_{\text {vib }}\left(S_{1}\right)<E_{\text {vib }}\left(T_{2}\right)<E_{\text {vib }}\left(T_{1}\right)$ we would expect the thresholds for ionization to be in the same order, $I_{\text {threshold }}\left(S_{1}\right)<I_{\text {threshold }}\left(T_{2}\right)<I_{\text {threshold }}\left(T_{1}\right)$. For a given probe energy (vertical arrow) $S_{1}, T_{2}$ and $T_{1}$ components of the excited state will be mapped onto different (but overlapping) portions of the photoionisation continuum.

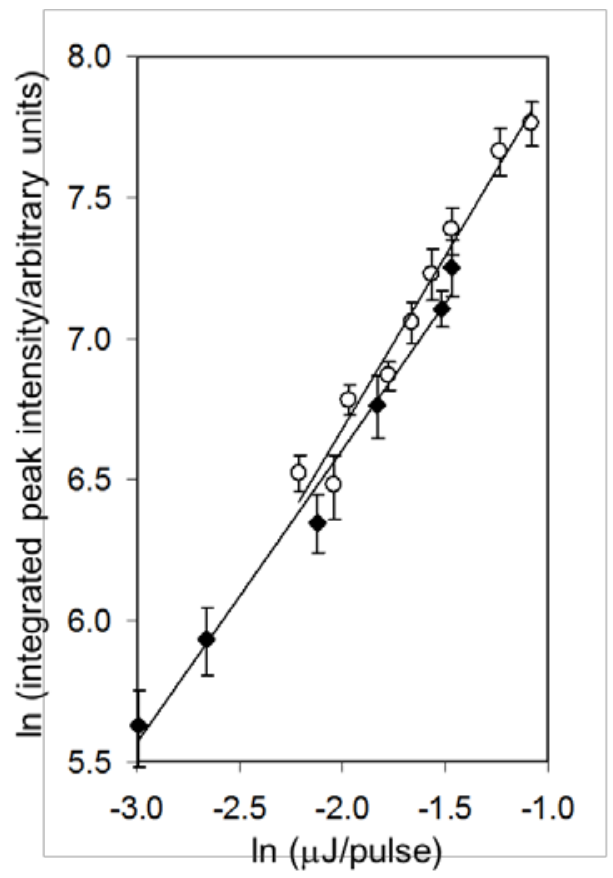

Figure 2

\section{Figure 2}

A plot of $\ln ($ integrated photoelectron intensity) against $\ln (\mu \mathrm{J} /$ pulse $)$ for pump pulses centered at $243 \mathrm{~nm}$ and probe pulses centred at $235 \mathrm{~nm}$ (open circles) and $254 \mathrm{~nm}$ (filled diamonds). These measurements were performed by recording the integrated photoelectron intensity for pump-only, probe-only and pump+probe. The signal intensity at a particular laser power was recorded for 2000 laser shots and the error bars represent two standard deviations from the mean signal value. The gradients are $n=1.2 \pm 0.2$ (235 $\mathrm{nm})$ and $n=1.0 \pm 0.1(254 \mathrm{~nm})$. The errors are twice the standard deviation for the fit. 


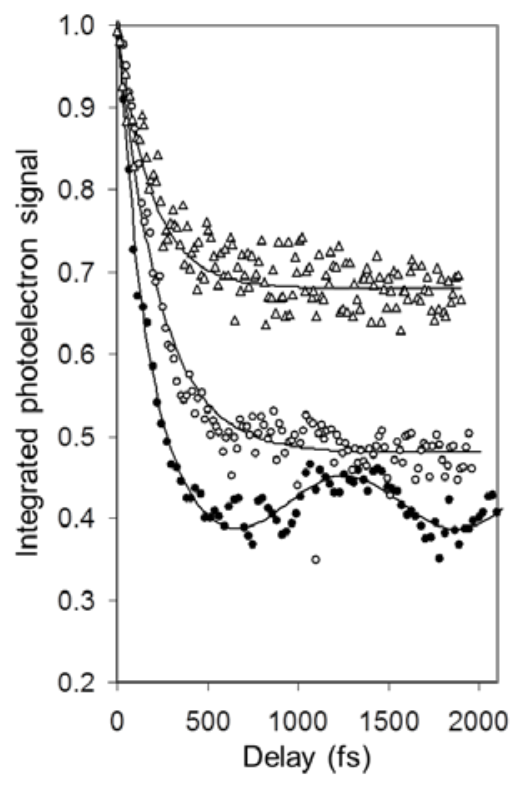

Figure 3

\section{Figure 3}

Experimental integrated photoelectron yield as a function of delay for probe wavelengths of $260 \mathrm{~nm}$ (filled circles), $254 \mathrm{~nm}$ (open circles) and $235 \mathrm{~nm}$ (triangles). The solid lines represent least squares fits to the observed data with functions described in the text (equations 2 and 4) and parameters listed in Table 1. The $260 \mathrm{~nm}$ probe can only ionize $\mathrm{S}_{1}$, the $254 \mathrm{~nm}$ probe can ionize $\mathrm{S}_{1}$ and $\mathrm{T}_{2}$ and the $235 \mathrm{~nm}$ probe can ionize $\mathrm{S}_{1}, \mathrm{~T}_{2}$ and $\mathrm{T}_{1}$.

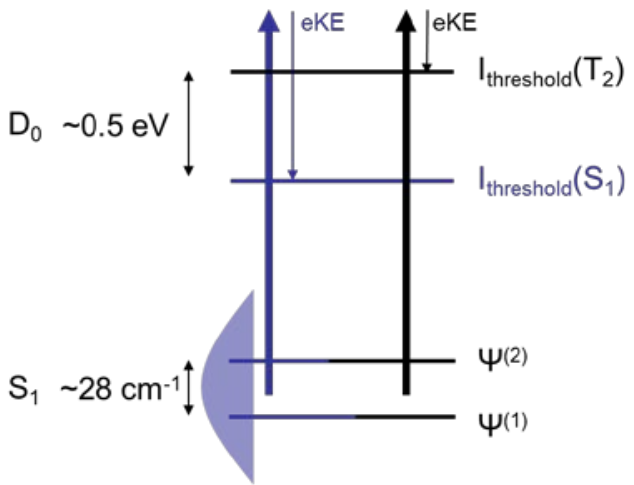

Figure 4 


\section{Figure 4}

Schematic diagram explaining the origin of the $1.2 \mathrm{ps}$ beat in the photoelectron signal. Components of the wave packet, $\psi^{(1)}$ and $\psi^{(2)}$, can each be written as a linear combination of "bright" and "dark" components. The ionization thresholds for these "bright" and "dark" components are separated by $\sim 0.5 \mathrm{eV}$. If the probe energy is below $I_{\text {threshold }}\left(T_{2}\right)$ then when population moves from the "bright" components to the "dark" components the integrated ionization signal drops. If the probe energy is above $I_{\text {threshold }}\left(T_{2}\right)$ then the integrated ionization signal does not depend on where the population is. In this case, the evolution of population between the "bright" and "dark" components can be monitored by measuring the time evolution of photoelectron kinetic energy distribution.

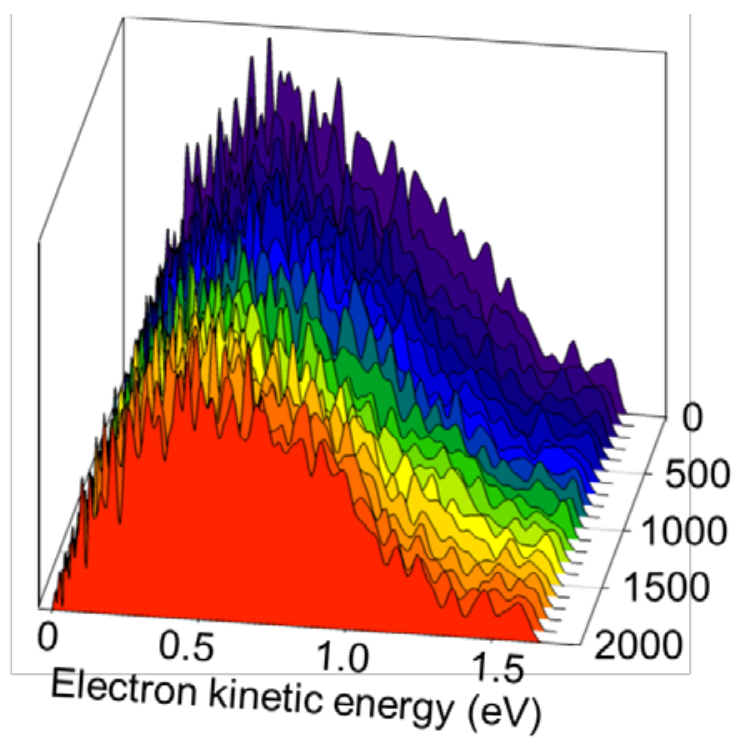

Figure 5

\section{Figure 5}

Photoelectron spectra recorded at 100 fs intervals following excitation of an effusive molecular beam of vibrationally hot benzene with a $243 \mathrm{~nm}$ pump pulse and a $235 \mathrm{~nm}$ probe pulse. 


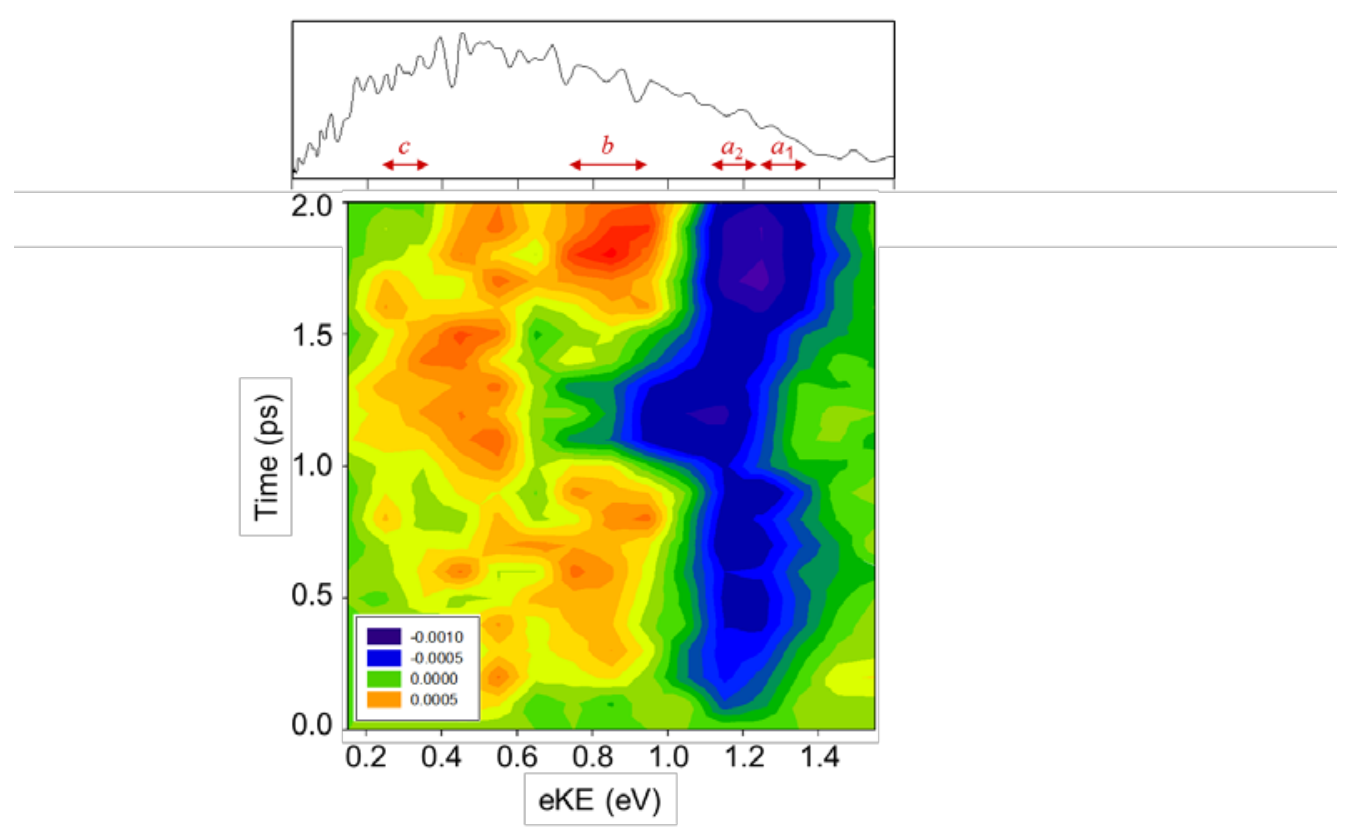

Figure 6

\section{Figure 6}

Difference-spectra: the individual photoelectron spectra in figure 5 are scaled so that they all have the same integrated area and the $t=0$ photoelectron spectra are subtracted from spectra recorded at all other pump-probe delays. Green represents no-difference, positive differences are represented by warm colours and negative differences by cold colours. The $t=0$ photoelectron spectra are plotted at the top of the difference-spectra.

Regions of interest highlighted by horizontal red arrows: 1.25-1.35 eV $\left(a_{1}\right)$; $1.13-1.23 \mathrm{eV}$ $\left(a_{1}\right) ; 0.75-0.95 \mathrm{eV}(b) ; 0.35-0.45 \mathrm{eV}(c)$. 


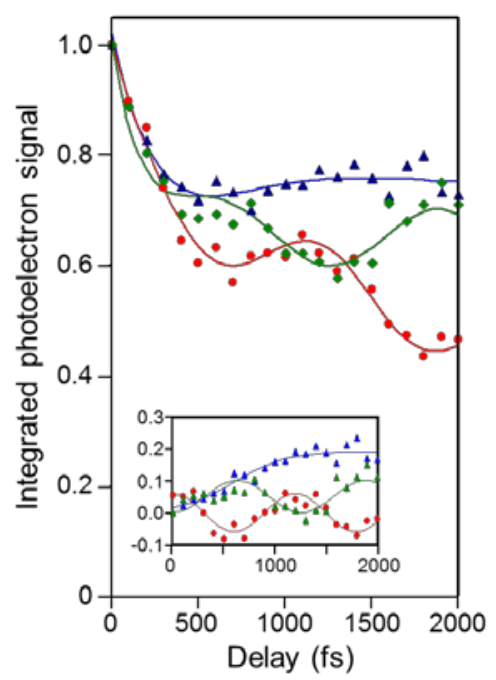

Figure 7

\section{Figure 7}

Experimental integrated photoelectron yields as a function of delay for a probe wavelength $243 \mathrm{~nm}$. Partial integrated photoelectron yields correspond to: 1.25-1.35 eV, $a_{1}$, $1.13-1.23 \mathrm{eV}, a_{2}$, (red circles); 0.75-0.95 eV, $b$, (green diamonds); 0.35-0.45 eV, $c$, (blue triangles).

Inset: partial integrated photoelectron yields with the biexponential decay removed. Symbols have the same meanings as above.

The solid lines in the main figure are fits to the partial integrated photoelectron yield. The fits are based on biexponential decays with added cosine, sine or error functions for the red, green and blue curves respectively. In all cases, the lifetimes of the fits match those given in Table 1. The fits in the inset correspond to the fits in the main figure with the biexponential components removed. 

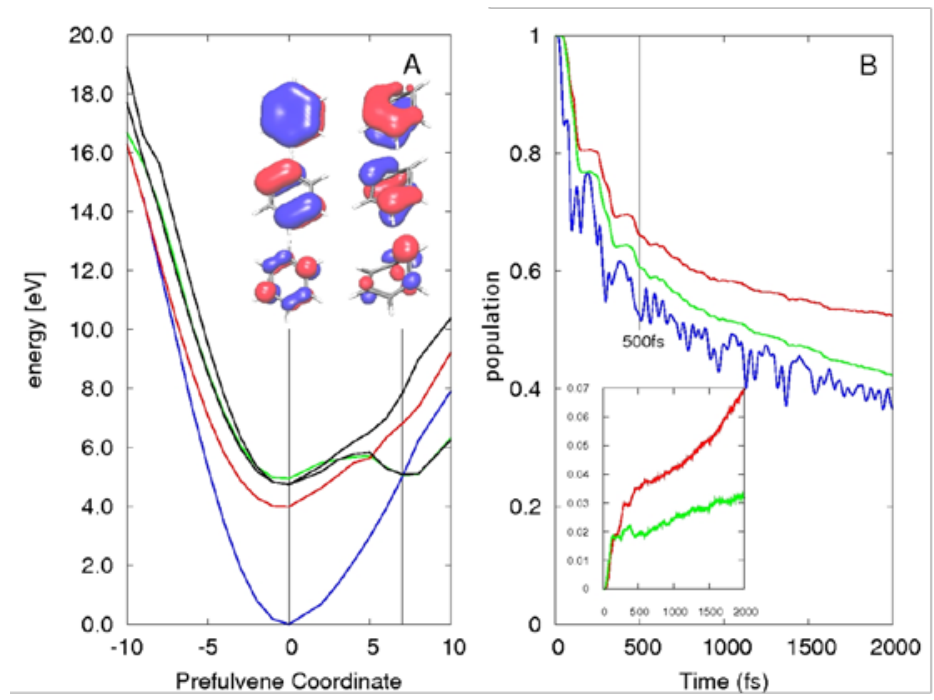

Figure 8

\section{Figure 8}

A. Cut through the potential energy surfaces of benzene along the "prefulvene" mode - a vector from the Franck-Condon point to the $\mathrm{S}_{1} / \mathrm{S}_{0}$ conical intersection - calculated using the MOLPRO program ${ }^{53}$ at the CASPT2 level with a $(6,6)$ CAS space and a Roos ANO basis set truncated to 6-31G* quality: $\mathrm{S}_{1}$ (green), $\mathrm{S}_{0}$ (blue), $\mathrm{T}_{1}$ (red) and $\mathrm{T}_{2}$ (black). The HOMO-1, HOMO and LUMO orbitals are shown at the Franck-Condon point and the $\mathrm{S}_{1} / \mathrm{S}_{0}$ conical intersection.

B. Relative diabatic state populations of benzene during the first 2000 fs following excitation into $S_{1}$. The blue line is the population of $S_{1}$ minus the population which is over the barrier to the $S_{1} / S_{0}$ intersection. This corresponds to the photoelectron data using the $254 \mathrm{~nm}$ probe wavelength. The green line is the population of the entire $\mathrm{S}_{1}$ population and the red line is the population of the $S_{1}$ plus triplet populations, corresponding to the experimental data recorded using the $235 \mathrm{~nm}$ probe pulse.

Inset: relative diabatic state populations for the triplet states only during the first $2000 \mathrm{fs}$ for the same calculations. The green line represents the $T_{2}$ population and the red line the $\mathrm{T}_{1}$ population. 


\section{References}

1 J. H. Callomon, T. M. Dunn and I. M. Mills, Philosophical Transactions of the Royal Society of London Series a-Mathematical and Physical Sciences, 1966, 259, 499.

2 J. H. Callomon, J. E. Parkin and Lopezdel.R, Chem. Phys. Lett., 1972, 13, 125.

3 J. H. Callomon and L. W. Somers, Chem. Phys. Lett., 1988, 144, 463.

4 M. Clara, K. Siglow and H. J. Neusser, Zeitschrift Fur Physikalische ChemieInternational Journal of Research in Physical Chemistry \& Chemical Physics, 2000, 214, 493.

5 C. E. Otis, J. L. Knee and P. M. Johnson, Journal of Chemical Physics, 1983, 78, 2091.

6 D. B. Moss and C. S. Parmenter, Journal of Physical Chemistry, 1986, 90, 1011.

7 C. E. Otis, J. L. Knee and P. M. Johnson, Journal of Physical Chemistry, 1983, 87, 2232.

8 E. Riedle, H. J. Neusser and E. W. Schlag, Journal of Physical Chemistry, 1982, 86, 4847.

9 E. Riedle, H. J. Neusser and E. W. Schlag, Faraday Discussions, 1983, 75, 387.

10 E. Riedle, H. J. Neusser and E. W. Schlag, Philosophical Transactions of the Royal Society of London Series a-Mathematical Physical and Engineering Sciences, 1990, 332, 189.

11 U. Schubert, E. Riedle, H. J. Neusser and E. W. Schlag, Journal of Chemical Physics, 1986, 84, 6182.

12 C. S. Parmenter and M. W. Schuyler, Chem. Phys. Lett., 1970, 6, 339.

13 W. R. Ware, B. K. Selinger, C. S. Parmenter and M. W. Schuyler, Chem. Phys. Lett., 1970, 6, 342.

14 K. G. Spears and S. A. Rice, Journal of Chemical Physics, 1971, 55, 5561.

15 L. Wunsch, H. J. Neusser and E. W. Schlag, Chem. Phys. Lett., 1975, 32, 210.

16 L. Wunsch, H. J. Neusser and E. W. Schlag, Chem. Phys. Lett., 1975, 31, 433.

17 G. H. Atkinson and C. S. Parmenter, Journal of Molecular Spectroscopy, 1978, 73, 20.

18 G. H. Atkinson and C. S. Parmenter, Journal of Molecular Spectroscopy, 1978, 73, 31.

19 G. H. Atkinson and C. S. Parmenter, Journal of Molecular Spectroscopy, 1978, 73, 52.

20 R. A. Coveleskie and C. S. Parmenter, Journal of Chemical Physics, 1978, 69, 1044.

21 C. S. Parmenter and K. Y. Tang, Chemical Physics, 1978, 27, 127.

22 M. Sumitani, D. Oconnor, Y. Takagi, N. Nakashima, K. Kamogawa, Y. Udagawa and K. Yoshihara, Chem. Phys. Lett., 1983, 97, 508.

23 T. A. Stephenson, P. L. Radloff and S. A. Rice, Journal of Chemical Physics, 1984, 81, 1060.

24 T. A. Stephenson and S. A. Rice, Journal of Chemical Physics, 1984, 81, 1073.

25 M. Sumitani, D. V. Oconnor, Y. Takagi, N. Nakashima, K. Kamogawa, Y. Udagawa and K. Yoshihara, Chemical Physics, 1985, 93, 359. 

Physics, 1987, 86, 2576.

27 R. J. Longfellow, D. B. Moss and C. S. Parmenter, Journal of Physical Chemistry, 1988, 92, 5438.

28 E. Riedle, T. Weber, U. Schubert, H. J. Neusser and E. W. Schlag, Journal of Chemical Physics, 1990, 93, 967.

29 M. Clara, T. Hellerer and H. J. Neusser, Applied Physics B-Lasers and Optics, 2000, 71, 431.

30 D. S. N. Parker, R. S. Minns, T. J. Penfold, G. A. Worth and H. H. Fielding, Chem. Phys. Lett., 2009, 469, 43.

31 I. J. Palmer, I. N. Ragazos, F. Bernardi, M. Olivucci and M. A. Robb, Journal of the American Chemical Society, 1993, 115, 673.

32 T. Suzuki, Annual Review of Physical Chemistry, 2006, 57, 555.

33 A. Stolow, A. E. Bragg and D. M. Neumark, Chemical Reviews, 2004, 104, 1719.

34 A. Stolow and J. G. Underwood, Advances in Chemical Physics, 139, 2008, 139, 497.

35 T. Suzuki and B. J. Whitaker, International Reviews in Physical Chemistry, 2001, 20, 313.

36 A. Assion, T. Baumert, J. Helbing, V. Seyfried and G. Gerber, Chem. Phys. Lett., 1996, 259, 488.

37 V. Blanchet, M. Z. Zgierski, T. Seideman and A. Stolow, Nature, 1999, 401, 52.

38 C. Z. Bisgaard, O. J. Clarkin, G. R. Wu, A. M. D. Lee, O. Gessner, C. C. Hayden and A. Stolow, Science, 2009, 323, 1464.

39 O. Gessner, A. M. D. Lee, J. P. Shaffer, H. Reisler, S. V. Levchenko, A. I. Krylov, J. G. Underwood, H. Shi, A. L. L. East, D. M. Wardlaw, E. T. Chrysostom, C. C. Hayden and A. Stolow, Science, 2006, 311, 219.

40 O. Gessner, E. T. H. Chrysostom, A. M. D. Lee, D. M. Wardlaw, M. L. Ho, S. J. Lee, B. M. Cheng, M. Z. Zgierski, I. C. Chen, J. P. Shaffer, C. C. Hayden and A. Stolow, Faraday Discussions, 2004, 127, 193.

41 C. J. Hammond, K. L. Reid and K. L. Ronayne, Journal of Chemical Physics, 2006, 124, 201102.

42 B. Kim, C. P. Schick and P. M. Weber, Journal of Chemical Physics, 1995, 103, 6903.

43 M. de Groot, R. W. Field and W. J. Buma, Proceedings of the National Academy of Sciences of the United States of America, 2009, 106, 2510.

44 S. R. Long, J. T. Meek and J. P. Reilly, Journal of Chemical Physics, 1983, 79, 3206.

45 A. Eppink and D. H. Parker, Review of Scientific Instruments, 1997, 68, 3477.

46 G. A. Garcia, L. Nahon and I. Powis, Review of Scientific Instruments, 2004, 75, 4989.

47 G. A. Worth, R. E. Carley and H. H. Fielding, Chemical Physics, 2007, 338, 220.

48 S. Aloise, C. Ruckebusch, L. Blanchet, J. Rehault, G. Buntinx and J. P. Huvenne, Journal of Physical Chemistry A, 2008, 112, 224.

49 J. S. Zugazagoitia, C. X. Almora-Diaz and J. Peon, Journal of Physical Chemistry A, 2008, 112, 358. 
50 S. Cogan, Y. Haas and S. Zilberg, Journal of Photochemistry and Photobiology aChemistry, 2007, 190, 200.

51 T. J. Penfold, R. S. Minns, D. S. N. Parker, H. H. Fielding and G. A. Worth, In Preparation, 2009.

52 T. J. Penfold and G. A. Worth, Journal of Chemical Physics, 2009, 131, 064303.

53 P. J. K. H.-J. Werner, R. Lindh, F. R. Manby, M. Schütz, P. Celani, T. Korona, A. Mitrushenkov, G. Rauhut, T. B. Adler, R. D. Amos, A. Bernhardsson, A. Berning, D. L. Cooper, M. J. O. Deegan, A. J. Dobbyn, F. Eckert, E. Goll, C. Hampel, G. Hetzer, T. Hrenar, G. Knizia, C. Köppl, Y. Liu, A. W. Lloyd, R. A. Mata, A. J. May, S. J. McNicholas, W. Meyer, M. E. Mura, A. Nicklaß, P. Palmieri, K.

Pflüger, R. Pitzer, M. Reiher, U. Schumann, H. Stoll, A. J. Stone, R. Tarroni, T. Thorsteinsson, M. Wang, A. Wolf . Molpro version 2008.1, a package of ab inition programs.

54 G. A. Worth, M. H. Beck, A. Jackle and H. D. Meyer, The Heidelberg MCTDH Package: A set of programs for multidimensional quantum dynamics, Version 8., 2000. 